\title{
Corpus
}

3 | 2004

Usage des corpus en phonologie

\section{L'analogie est-elle un fait fonctionnel ou grammatical ? Le cas de l'apophonie portugaise}

Joaquim Brandăo de Carvalho

\section{OpenEdition}

1 Journals

Édition électronique

URL : http://journals.openedition.org/corpus/194

DOI : $10.4000 /$ corpus. 194

ISSN : 1765-3126

Éditeur

Bases; corpus et langage - UMR 6039

Édition imprimée

Date de publication : 1 décembre 2004

ISSN : 1638-9808

Référence électronique

Joaquim Brandăo de Carvalho, "L'analogie est-elle un fait fonctionnel ou grammatical ? Le cas de l'apophonie portugaise », Corpus [En ligne], 3 | 2004, mis en ligne le 02 décembre 2005, consulté le 08 septembre 2020. URL : http://journals.openedition.org/corpus/194; DOI : https://doi.org/10.4000/ corpus. 194 


\title{
L'analogie est-elle un fait fonctionnel ou grammatical ? Le cas de l'apophonie portugaise
}

\author{
Joaquim BRANDÃO DE CARVALHO \\ UMR 7023, Université Paris 8
}

Résumé : Il est couramment admis qu'il existe une corrélation, sinon un rapport de cause à effet, entre la fréquence lexicale d'une dérivation et sa productivité, mesurée à l'aune de sa force d'attraction analogique. Or cette thèse est démentie par l'examen détaillé des faits d'apophonie nominale en portugais. De deux alternances qui se partagent un sous-ensemble de nominaux, c'est la dérivation minoritaire, ainsi que le révèle l'analyse d'un corpus de 238 mots, qui, depuis le XIX ${ }^{\text {ème }}$ siècle, gagne lentement du terrain dans le standard de Lisbonne, et y exerce une force analogique sur l'alternance majoritaire sans commune mesure avec le phénomène inverse. On déduira de cet apparent paradoxe et de sa spécificité dialectale que le véritable moteur du changement analogique ne réside pas dans le nombre d'entrées voire de sorties lexicales, mais dans l'existence, au sein de la grammaire du locuteur, de "schèmes dérivationnels" pouvant à l'occasion être mis en concurrence sous certaines conditions distributionnelles et sociolinguistiques.

\section{Introduction}

Que nous apprennent les corpus sur la productivité d'une alternance ? Y a-t-il corrélation entre la fréquence lexicale d'une dérivation et sa productivité, mesurée à l'aune de sa force d'attraction analogique ? Telles sont les questions que pose l'examen détaillé des faits d'apophonie nominale en portugais. De deux alternances qui se partagent un sous-ensemble de nominaux, c'est la dérivation minoritaire, ainsi que le révèle l'analyse d'un corpus de 238 mots, qui, depuis le XIX ${ }^{\text {ème }}$ siècle, gagne lentement du terrain dans le standard de Lisbonne, et y exerce une force analogique sur l'alternance majoritaire sans commune mesure avec le phénomène inverse. On déduira de cet 


\section{J. BRANDÃO DE CARVALHO}

apparent paradoxe que le moteur du changement analogique ne réside pas dans le nombre d'entrées ou de sorties lexicales, mais dans l'existence de "schèmes dérivationnels " potentiellement concurrents au sein de la grammaire sous certaines conditions distributionnelles et sociolinguistiques.

\section{Les faits}

En portugais, les noms et adjectifs paroxytons singuliers masculins en - $O$ comportant un $/ \mathrm{o} /$ accentué se divisent en deux classes selon le type de dérivation de leurs pluriels (plus, le cas échéant, de leurs féminins, singuliers et pluriels). Dans les deux cas, $-s$ s'ajoute au radical (et $-a(s)$ se substitue à $-o$ ) ; dans l'un, cependant, la voyelle accentuée ne change pas, alors qu'elle devient $/ \mathrm{o} /$ dans l'autre :

$$
\text { Sing. masc. Pluriel et féminin }
$$
a.

$$
\begin{array}{ll}
\text { o } & \rightarrow \\
\text { 1[o]bo } & \rightarrow \\
\text { "loup" } &
\end{array}
$$$$
\text { o }
$$$$
1[\mathrm{o}] \mathrm{bos}, 1[\mathrm{o}] \mathrm{ba}(\mathrm{s})
$$$$
\text { "loups", "louve(s)" }
$$
b.$$
\begin{array}{lll}
\mathrm{o} & \rightarrow & 0 \\
\mathrm{p}[\mathrm{o}] \mathrm{rco} & \rightarrow & \mathrm{p}[\mathrm{o}] \mathrm{rcos}, \mathrm{p}[\mathrm{o}] \mathrm{rca}(\mathrm{s}) \\
\text { "porc" } & & \text { "porcs", "truie(s)" }
\end{array}
$$

La seconde dérivation est dite " apophonique ».

C'est là un phénomène en tous points lexical: d'une part, et contrairement à la métaphonie typique des conjugaisons verbales portugaises ( $c f$. Carvalho 2004), l'alternance (1b) est, comme on le verra, minoritaire dans le lexique concerné ; d'autre part, et surtout, nul objet phonologique n'affleure dans la morphologie du pluriel qui eût pu expliquer l'ouverture de la voyelle, à l'inverse, là encore, de la métaphonie verbale, dont les responsables - les voyelles thématiques - font surface dans la majorité des formes.

Comme tous les phénomènes de ce type, l'apophonie et son absence ne s'expliquent phonologiquement qu'au plan diachronique. Le /o/ de la dérivation (1a) remonte à lat. vulg.

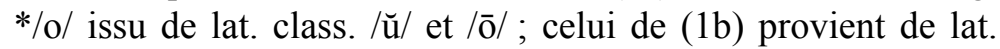
vulg. */o/ issu de /ŏ/. Il suit que, des quatre voyelles de (1), c'est 
la voyelle accentuée du masculin singulier en (1b) qui a changé par rapport au latin vulgaire. Les grammaires historiques du portugais (Huber 1933: § 93.2; Williams 1938: § 123.6) s'accordent à y voir un effet métaphonique sur $* / \mathrm{s} /$ de la terminaison classique /-ŭ/, harmonie fermante qui remonterait donc à une époque où cette désinence se différenciait encore de celle du pluriel /-ōs/ : ${ }^{1}$

$$
\begin{aligned}
& \text { a. } \quad \text { Lat. vulg. */o/ }>[\mathrm{o}] / \ldots\left(\mathrm{C}^{*}\right) \mathrm{u} \\
& \text { b. } * \mathrm{p}[\mathrm{o}] \mathrm{rc}[\mathrm{u}]>* \mathrm{p}[\mathrm{o}] \mathrm{rc}[\mathrm{u}]>\mathrm{p}[\mathrm{o}] \mathrm{rco} \\
& \text { mais } * \mathrm{p}[\mathrm{o}] \mathrm{rc}[\mathrm{o}] \mathrm{s}>* \mathrm{p}[\mathrm{o}] \mathrm{rc}[\mathrm{o}] \mathrm{s}>\mathrm{p}[\mathrm{o}] \mathrm{rcos}
\end{aligned}
$$

Dès lors qu'il y eut confusion des timbres à la finale, la métaphonie, jusque là conditionnée par le contexte, devint apophonie, mutation imprévisible sur une base phonique, et l'alternance, rendue opaque, se trouva désormais exposée au jeu de l'analogie. ${ }^{2}$

La connaissance de l'origine phonétique du phénomène nous fournit cependant un "marqueur » permettant d'évaluer les dérives postérieures : seront ainsi, en principe ( $c f$. § 3.2), réputées analogiques les deux classes lexicales en (3):

(3) Formes analogiques:

(a) Mots dont le /o/ ne provient pas de $* / \mathrm{o} /$, mais qui font leur pluriel en $/ \mathrm{o} /$;

(b) Mots dont le $/ \mathrm{o} /$ provient de $* / \mathrm{s} /$, mais qui font leur pluriel en $/ \mathrm{o} /$.

1. Lat. vulg. */ع/ a dû amorcer une dérive analogue dont il ne reste cependant que peu de traces : $c f$., par exemple, port. $m[\mathrm{e}] d o$ " peur » $\langle$ lat. métu $\rangle$ esp. miedo; le pluriel en est cependant $m[\mathrm{e}] d o s$.

2. Je me cantonnerai ici à la pluralisation. Il n'est pas absurde de soutenir que le $/ \mathrm{o} / \mathrm{du}$ féminin a pu être réinterprété par les locuteurs comme une métaphonie induite par $-a$, dont certaines évolutions de lat. vulg. */o/ (cf. forma, hora $>f[0] \mathrm{rma}, h[\mathrm{o}] \mathrm{ra}$ ) et */e/ (cf. ista, illa $>[\varepsilon] s t a,[\varepsilon] l a)$ apporteraient la preuve, et qui est confortée par la métaphonie verbale du $1^{\text {er }}$ groupe ( $c f$. Carvalho 2004). Une thèse similaire a été défendue par Russo (2001) pour le napolitain, qui témoigne d'une apophonie voisine. 


\section{J. BRANDÃO DE CARVALHO}

Plus étendu est l'ensemble ( $3 a$ ) au sein de la classe des mots à apophonie, plus forte sera l'analogie exercée par l'alternance apophonique; à l'inverse, plus fourni est l'ensemble (3b) parmi les formes non apophoniques, plus élevée sera la force d'attraction de ces dernières.

Comme on peut s'en douter - la voyelle n'ayant pas subi le changement en (2), i.e. lat. vulg. */o/, remontant à deux phonèmes classiques $(/ \breve{\mathrm{u}} /$ et $/ \overline{\mathrm{o}} /)$, alors que celle qui l'a subi ne provient que d'un seul (/o/) -, les formes non apophoniques sont majoritaires en portugais. Cependant, leur prédominance fut naguère encore plus forte que ne le laisserait à penser la seule étymologie, ce qui révèle a contrario une progression récente des alternances apophoniques: selon Nunes (1945: 231, n. 2), «[...] jusqu'à la fin du XVIII ${ }^{\text {ème }}$ siècle, comme en général encore de nos jours ${ }^{3}$ parmi le peuple, conservaient au pluriel le o fermé du singulier [...] poços, ossos, ovos, corpos, canhotos, tortos, etc. ». Sur ces six exemples, les cinq derniers auraient dû être apophoniques et se prononcer avec $/ \mathrm{d} /$; ils le sont effectivement dans la norme moderne de Lisbonne, à l'exception de canhotos "gauchers ", et témoignent ainsi d'un retour en force de l'apophonie, qui s'est par ailleurs annexé le premier mot cité par Nunes, poços «puits », historiquement non apophonique (< lat. pŭtěu). Si l'état de langue auquel se réfère Nunes reflétait une tendance naturelle à la généralisation de la dérivation majoritaire, alors ces quelques exemples suggèrent l'hypothèse d'un renversement de la tendance analogique au profit cette fois de l'alternance apophonique pourtant minoritaire. C'est ce que je me suis efforcé de vérifier à travers l'analyse du lexique portugais.

\section{Analyse}

\subsection{Corpus}

Le corpus est constitué de 238 noms et adjectifs masculins singuliers paroxytons ayant /o/ comme voyelle accentuée et $-o$ comme voyelle finale. Une première liste d'environ 150 mots, collectée dans le Dicionário da língua

3. La première édition du Compêndio de Nunes date de 1919. 


\section{L' analogie est-elle un fait fonctionnel ou grammatical?}

portuguesa contemporânea da Academia das Ciências de Lisboa (= DLPC 2001), a ensuite été complétée et remaniée en fonction des données fournies par une version électronique du Mini-dicionário Aurélio (= Ferreira 1977) ${ }^{4}$.

Cette liste de 238 entrées lexicales a été obtenue après élimination des items qui posaient problème, soit que le mot parût trop spécialisé (terme technique ou régional), soit, dans neuf cas d'étymologie inconnue, qu'il ne fût pas possible d'en déterminer le caractère analogique ou non du pluriel. D'autre part, pour des raisons qui seront exposées en $\S 3.2$, deux types de formes ont été exclus du corpus: (a) les mots où $o$ est nasalisé ou suivi d'une consonne nasale hétérosyllabique ; (b) les nombreux adjectifs en -oso, cette désinence constituant une entrée unique ; c'est là, d'ailleurs, le seul affixe du corpus.

Le classement des 238 mots dans chacune des classes apophonique et non apophonique, d'après la voyelle accentuée de leur pluriel, reflète l'usage standard (língua padrão) de Lisbonne, tel qu'il ressort du $D L P C$, de mon propre parler et de celui de deux autres locuteurs à qui le corpus a été soumis. Il s'agit donc d'un dialecte particulier, la variation dialectale n'étant pas le sujet de cette étude. Aussi n'ai-je pas tenu compte des cas de variation affectant de nombreux mots, qui distinguent l'usage standard européen d'autres dialectes, y compris, sans doute, lisboètes, mais surtout septentrionaux et brésiliens. Je dirai simplement là-dessus, après des sondages portant sur un petit nombre d'items, que le stade linguistique évoqué par Nunes semble s'être plus largement maintenu en province (et au Brésil), car bien plus nombreux sont les cas où le standard de Lisbonne présente des pluriels en $/ \mathrm{o} /$ face à des formes non standard (ou brésiliennes) en $/ \mathrm{o} /$ que les cas où le premier a $/ \mathrm{o} /$ correspondant à des pluriels en $/ \mathrm{\jmath} /$ ailleurs.

4. Cette version électronique, dénommée Listas, comprend 27074 entrées et a été élaborée au Laboratório de Fonética e Psicolingüística (LAFAPE) de l'Université d'Etat de Campinas (UNICAMP), São Paulo. Je remercie vivement sa directrice, Eleonora Cavalcante Albano, qui m'a aimablement envoyé, après sélection à l'aide de ce programme, le lexique pertinent pour mon analyse. 


\section{J. BRANDÃO DE CARVALHO}

\subsection{Méthodologie}

Quelques nuances et précautions de méthode sont à apporter à la définition générale donnée en (3) des formes analogiques. S'agissant de la partie la plus ancienne du lexique, pour déterminer si tel ou tel mot a connu ou non un changement analogique, je me suis servi de deux critères fondamentaux : par ordre de priorité,

(4) a. la correspondance avec le castillan (voire avec d'autres langues romanes) ;

b. l'étymologie latine.

Le recours à (4a) s'explique aisément. Il existe, en particulier, de nombreux mots à étymologie inconnue communs aux deux langues péninsulaires dont seule la forme espagnole, compte tenu à la fois de l'absence d'apophonie en castillan et de la diphtongaison bien connue de $* / \mathrm{o} /$ accentué en ue dans cette langue, peut nous fournir la proto-voyelle radicale, selon le schéma :
a. Cast. $/ \mathrm{o} /{ }^{\circledR} * / \mathrm{o} /$
Ex. : mofo, moço, etc.
b. Cast. /we/ $® * / 2 /$
Ex. : pescoço, soro, etc.

Corollaire de la primauté de (4a) sur (4b), si le castillan ou les correspondances intra-romanes impliquent un étymon dont la voyelle ne correspond pas à ce qu'on trouve en latin classique, c'est la forme vulgaire reconstruite qui a servi à juger $\mathrm{du}$ caractère analogique ou non analogique du mot portugais. Par exemple, cast. huevo et fr. euf, qui présupposent */o/, indiquent que port. [0]vos est étymologique, en dépit d'une forme classique ōvos qui aurait dû donner *[o]vos; de même, cast. Godo "Goth » implique que port. G[o]dos est, lui aussi, étymologique, quoiqu'on se soit attendu à * $G[0] d o s$ en vertu de lat. Göthos.

Il va de soi, d'autre part, qu'il faut ajouter aux critères édictés en (4) ce que l'on connaît de la phonologie diachronique du portugais et de l'ibéro-roman en général. Ainsi, quand bien même la correspondance entre castillan et portugais et/ou l'étymologie latine impliqueraient un $* / \mathrm{o}$ / pour un mot subissant 


\section{L' analogie est-elle un fait fonctionnel ou grammatical?}

l'apohonie, je ne l'ai considéré comme non analogique que si celle-ci ne contrevient pas aux changements phonétiques qui auraient dû fermer $* / \mathrm{o} /$ en $/ \mathrm{o} /$, tant au singulier qu'au pluriel. Tel est le cas de la dilation induite par palatale. Par exemple, almoço "déjeuner » ou pescoço « cou », qui font leur pluriel en $/ \mathrm{o} /$, remontent à une forme en $* / \mathrm{o} /$ compte tenu de cast. almuerzo et pescuezo; ces pluriels doivent pourtant être vus comme analogiques, l'élément palatal jadis présent dans la dernière syllabe ayant conduit à la fermeture de $* / \mathrm{o} /$ en portugais, comme le montre la comparaison entre $f[\mathrm{o}] r c ̧ a$ et cast. fuerza. Il en va de même, à plus forte raison, lorsque seule l'étymologie latine ou des cognats plus lointains supposent un */o/, la fermeture induite par palatale ayant eu lieu tant en castillan qu'en portugais : ainsi, [0]lhos « yeux » (cp. cast. ojos) est analogique, malgré lat. ǒculu et fr. oxil.

La possibilité de changements analogiques devant ancienne palatale implique que l'opposition /o/: / $/$ existe, dans ce contexte, dans la langue moderne, et que la fermeture induite par la palatale n'est plus un changement «actif». Beaucoup moins clair, cependant, est le cas des mots où une consonne nasale hétérosyllabique suit la voyelle accentuée. Ici, suite à une ancienne nasalisation, les oppositions $/ \mathrm{e} \mathrm{o} /: / \varepsilon \mathrm{o} /$ ont été longtemps neutralisées au profit du timbre mi-fermé [e o], et le sont toujours dans la plupart des usages brésiliens. Un rétablissement est en cours dans la língua padrão européenne, comme le montrent les effets encore très variables de la métaphonie verbale du $1^{\text {er }}$ groupe ( $c f$. Carvalho 2004). ${ }^{5}$ Parmi les noms et adjectifs, même si / $/ \varepsilon /$ et $/ \mathrm{J} /$ ont parfois été restaurés sous l'effet d'influences savantes latinisantes, ${ }^{6}$ aucun mot ne subit d'apophonie : ainsi, dono «maître », sono « sommeil»,

5. Ainsi a-t-on $t[0] m o$ " je prends », mais $s[0] n h o$ " je rêve »; $c f$. aussi les hésitations entre $r[\varepsilon] m o$ et $r[\mathrm{e}] m o$ " je rame», eng[o]mo et eng[o]mo « je repasse », ord $[\varepsilon]$ no et $\operatorname{ord}[\mathrm{e}]$ no «j'ordonne », $a b[0]$ no et $a b[\mathrm{o}]$ no « je garantis $»$, etc.

6. Etymologiquement correctes (par exemple, $h[0]$ mem «homme» de lat. hŏmine, dem[o]nio « démon » de daemŏnĭu) ou non ( $c f . r[\varepsilon] m o$ « rame » pour lat. rēmu, Ant[0]nio «Antoine» pour Antōniu, c[o]mico « comique » pour $\mathrm{commicu}$ ). 


\section{J. BRANDÃO DE CARVALHO}

sonho 《rêve », tomo «tome", par exemple, font tous leur pluriel en /o/, malgré le $\breve{o}$ de leurs étymons ( $c f$. cast. dueño, sueño). Face à un changement manifestement en cours, et devant la possibilité d'un conditionnement phonologique au moins partiel de l'alternance /o/ - /o/ devant nasale, j'ai choisi d'exclure du corpus les (30) mots concernés, puisqu'ils auraient gonflé indûment le nombre de formes analogiques de la classe non apophonique. ${ }^{7}$ Il en va de même, a fortiori, des mots où voyelle et nasalité sont tautosyllabiques, tels conto " conte", tronco "tronc», lombo "filet», etc. : celle-ci implique une stricte neutralisation des distinctions $/ \mathrm{e} o /: / \varepsilon$ o/ dans la lingua padrão. ${ }^{8}$

A noter, enfin, que j'ai traité de façon unitaire les 283 adjectifs en -oso «-eux» recensés dans Ferreira (1977): hautement productive, cette désinence est représentée dans le corpus par le seul item -oso, ${ }^{9}$ conformément à l'hypothèse, discutée en $\S 4$, selon laquelle les membres d'une classe ouverte ne sont pas stockés dans le lexique.

\subsection{Résultats}

La Figure 1 illustre la répartition des 238 mots du corpus dans les classes apophonique (CAp) et non apophonique

7. Ce choix présente, de plus, l'avantage de faciliter la comparaison éventuelle des résultats obtenus pour le standard européen avec des données brésiliennes, où l'apophonie est indubitablement impossible devant nasale hétérosyllabique, du moins dans la norme fondée sur le parler de Rio de Janeiro. Une telle comparaison ferait ressortir la spécificité de l'évolution lisboète.

8. C'est pour une raison similaire que les mots ayant ou comme voyelle accentuée n'ont pas été non plus pris en compte. Cette ancienne diphtongue ne subsiste que dans le nord du Portugal et s'est contractée ailleurs en [o], lequel ne subit jamais d'apophonie. Cependant, la monophtongaison est si récente et le comportement de ou si particulier - alternant parfois avec oi et ne se réduisant pas, au Portugal, à la prétonique - qu'il est, là encore, préférable d'imputer l'absence d'alternance [o] - [0] dans ces mots à des facteurs phonologiques : il s'agit, en l'occurrence, d'un phonème distinct (cf. Carvalho 1994).

9. Il en va de même des divers dérivés en -posto, de pôr "mettre, poser », ramenés à la seule entrée -posto (hormis posto «poste» et imposto « impôt»), et de doublets du type porto / aeroporto / heliporto, demagogo / pedagogo, filantropo / misantropo... 


\section{L' analogie est-elle un fait fonctionnel ou grammatical?}

(CNAp), ainsi que le nombre de formes analogiques (FAn) et non analogiques (FNAn) dans chacune de ces classes. (La liste complète des items est fournie en annexe.)

On le voit, les deux dérivations se partagent à parts égales l'ensemble des formes analogiques. Cette égalité doit cependant être appréciée en regard de leur taille respective. La Figure 2 montre en vis-à-vis la part des deux classes apophonique et non apophonique dans le corpus total, ainsi que le pourcentage de formes analogiques au sein de chacune d'entre elles.

FIG. 1

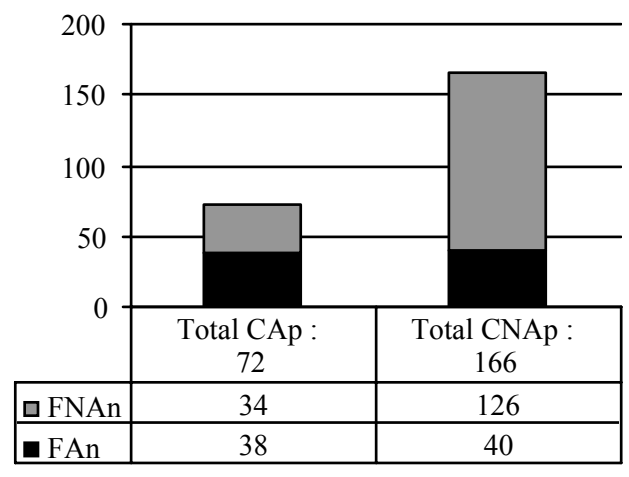

FIG. 2

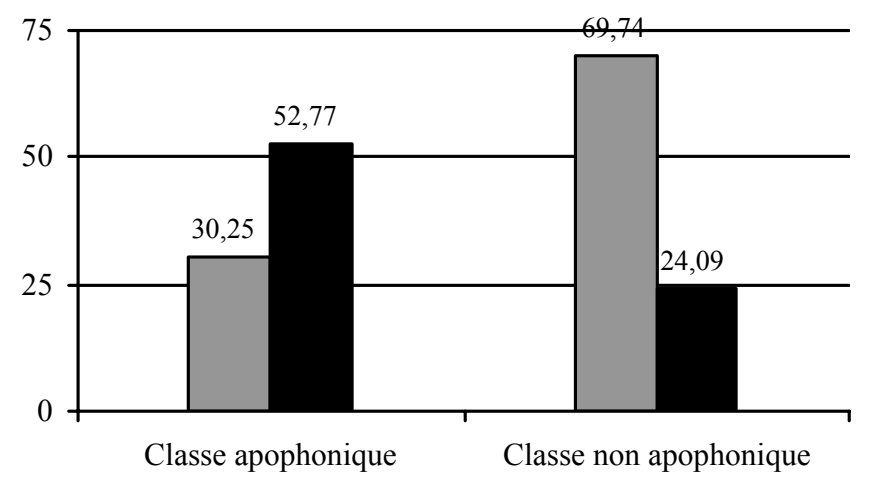

․ Part de la classe dans le corpus (en \%)

- Taux d'analogie au sein de la classe (en \%) 


\section{J. BRANDÃO DE CARVALHO}

Dans le Tableau 1, le test du khi-deux $\left(\left.\right|^{2}\right)$ précise la signification statistique du pourcentage de FAn dans la classe apophonique à travers la comparaison des effectifs observés avec les effectifs attendus pour les quatre ensembles d'items de la Figure $1:{ }^{10}$

\begin{tabular}{|c|c|c|c|c|c|c|}
\hline \multicolumn{2}{|c|}{ TABLEAU 1} & CAp & $\%$ & CNAp & $\%$ & Total \\
\hline \multirow{2}{*}{$\begin{array}{l}\text { Résultats } \\
\text { observés }\end{array}$} & FNAn & 34 & 14,28 & 126 & 52,94 & 160 \\
\hline & FAn & 38 & 15,96 & 40 & 16,80 & 78 \\
\hline \multirow{2}{*}{$\begin{array}{l}\text { Résultats } \\
\text { attendus }\end{array}$} & FNAn & 48,40 & 20,33 & 111,59 & 46,88 & 160 \\
\hline & FAn & 23,59 & 9,91 & 54,40 & 22,85 & 78 \\
\hline \multicolumn{2}{|l|}{ Total } & 72 & 30,25 & 166 & 69,74 & 238 \\
\hline \multicolumn{2}{|c|}{ Signification } & \multicolumn{3}{|c|}{$\left.\right|^{2}=18,75$} & \multicolumn{2}{|c|}{$\overline{p<<0,0001}$} \\
\hline
\end{tabular}

La valeur de $\left.\right|^{2}(18,75$ pour un seuil de probabilité $p<0,0001)$ est hautement significative de ce que l'attraction analogique exercée par l'alternance apophonique est supérieure à celle exercée par l'autre dérivation, et ce en dépit du caractère minoritaire de la première dans le lexique.

\section{Problématique}

Selon Hock (1988 : 173), qui offre une bonne synthèse des vues couramment admises en la matière, la fréquence d'une alternance « may be more a symptom than a cause of productivity ». Le bon sens voudrait peut-être qu'il y ait au moins une «interaction» entre fréquence et productivité telle qu'une dérivation productive regroupe naturellement plus d'items lexicaux qu'une dérivation moins productive, ce qui, à son tour, conforte la productivité de celle-là. Mais on peut aussi craindre que, par un renversement épistémologique qui a des précédents en phonologie (songeons, par exemple, au rapport entre

10. Pour trouver l'effectif attendu (E) dans chaque cellule du tableau, on applique la formule suivante (où $\mathrm{T}=$ total, $\mathrm{L}=$ ligne et $\mathrm{C}=$ colonne) : $\mathrm{E}$ $=\left(\mathrm{T}_{\mathrm{L}} \times \mathrm{T}_{\mathrm{C}}\right) / \mathrm{T}$. 


\section{L' analogie est-elle un fait fonctionnel ou grammatical?}

《 règles » et « régularités »), le terme « interaction », appliqué à une « corrélation » entre deux phénomènes observés, ne cache en fait une pure circularité au niveau explicatif, la productivité d'une alternance pouvant aussi aisément s'expliquer par sa fréquence que, réciproquement, celle-ci par celle-là. Cherchons donc des critères de la productivité ailleurs que dans la fréquence lexicale.

Le traitement de la plupart des emprunts relevés dans le corpus, par exemple, conduit certes à faire de l'absence d'apophonie majoritaire la dérivation la plus productive: si desp [0]rto « sport» donne bien desp[0]rtos, loto « loto», risoto « risotto », golo «but» (au football) (< angl. goal), etc. font leurs pluriels en $/ \mathrm{o} /$. Toutefois, un autre critère non moins satisfaisant, d'ordre sémantique, ne fournit pas de réponse claire ici. Des formes anglaises telles que *beek et *spice pour books et spouses sont sémantiquement opaques à l'inverse de foots et mouses pour feet et mice; il suit que c'est la suffixation de $-s$ qui constitue, en anglais, la dérivation productive du pluriel ( $c f$. Hock 1988 : 174). Or, en portugais, la dérivation apophonique serait tout aussi compréhensible pour le locuteur que sa contrepartie non apophonique, et ce quel que soit le mot. Le critère sémantique indique donc simplement que les deux alternances sont productives.

C'est, en effet, ce que confirme l'analyse du corpus menée en $\S 3$, qui dément du coup le présupposé couramment admis et énoncé ci-dessus, puisqu'une alternance pourtant statistiquement minoritaire est toujours (et, étant donné ce qui a été dit en $\S 2$, de plus en plus) productive, compte tenu du pourcentage de formes analogiques. Encore faut-il souligner que la force d'attraction de la classe apophonique est sans doute sous-estimée dans les statistiques données en $\S 3.3$. D’une part, le résultat du test du khi-deux dans le Tableau 1 est très probablement à majorer: il repose sur l'hypothèse selon laquelle le taux d'analogie devrait être constant, car proportionnel à la taille de chaque classe dans le corpus ; or, s'il existe une corrélation entre la productivité d'une alternance et sa fréquence lexicale, il ne s'ensuit pas qu'elles entretiennent un rapport linéaire. D'autre part, et surtout, compte tenu de la tendance à la disparition de l'apophonie ayant prévalu jusqu'à 


\section{J. BRANDÃO DE CARVALHO}

la fin du XVIII ${ }^{\text {ème }}$ siècle $(c f . \S 2)$, il est très probable, pour ne pas dire certain, que de nombreux cas d'alternance [o] - [0] considérés ici comme étymologiques, sur la base des cognats castillans et/ou de la voyelle latine, soient en réalité analogiques, l'apophonie n'y étant que l'effet d'une restauration moderne toujours inconnue de nombreuses variétés provinciales et brésiliennes ( $c f . \S 3.1$ ). C'est d'ailleurs ce qui ressort de la comparaison des données fournies par une locutrice avec les résultats exposés en $\S 3.3$ et détaillés en annexe, lesquels ne constituent qu'une sorte de moyenne pondérée en fonction des indications, relativement conservatrices, du DLPC (2001) : chez elle, après élimination des items dont le pluriel lui parait incertain, la classe apophonique comprend jusqu'à $44,3 \%$ des mots restants contre $30,25 \%$ ( $c f$. Fig. 2) ; or les formes jugées analogiques selon les critères retenus ne représentent que $60,6 \%$ de ce différentiel.

Toutefois, ce que nous révèlent d'intéressant les données portugaises n'est pas le fait en soi qu'une alternance minoritaire puisse être productive, mais qu'elle en vienne à concurrencer l'alternance majoritaire. L'alternance anglaise sing / sang / sung est, à l'instar de la dérivation apophonique portugaise, minoritaire - prétérit et participe se formant régulièrement et massivement par suffixation: RAD+ed - et, toujours comme la dérivation apophonique portugaise, productive - bring / brought / brought, par exemple, étant parfois analogiquement ramenés à bring / brang / brung, alors que l'on n'a jamais, à l'inverse, sing / *sought / *sought. Il s'agit ici, toutefois, d'une productivité relative, dont le domaine lexical fait de l'alternance sing / sang / sung une «régularité locale »: en ce sens, cette alternance-là reste majoritaire, en anglais, dans le sous-ensemble des verbes en -ing. Tel est aussi le cas des formes enfantines françaises comme prendu pour pris : parmi les verbes du $3^{\text {ème }}$ groupe, le premier participe est plus fréquent, et partant plus productif, que le second. Peu importe alors que *prendé, tout comme *singed, eussent représenté un type de flexion plus général : tout se passe comme si les processus analogiques avaient lieu dans un système de cercles concentriques où une sorte de " principe d'adjacence » leur interdit de sauter des étapes; on se sert du modèle général 


\section{L' analogie est-elle un fait fonctionnel ou grammatical?}

le plus proche. Or ce qui caractérise les faits portugais est que les alternances apophoniques n'y constituent même pas une « régularité locale » comparable à sing / sang / sung ou vendre / $v e n d u$, puisqu'elles restent minoritaires au sein du sousensemble des paroxytons masculins en - $o$ et à /o/ accentué ; aussi ne disposent-elles pas, à l'inverse des exemples anglais et français ci-dessus, d'une «niche morphologique » qui aurait pu les abriter et les préserver de l'élimination; pourtant, non seulement ces alternances apophoniques ont survécu jusqu'à aujourd'hui malgré leur absence de protection, mais encore elles ne sont pas moins productives, dans la langue moderne, que les dérivations non apophoniques statistiquement majoritaires au sein du même domaine lexical.

Comment une alternance minoritaire réussit-elle donc à se maintenir, voire à s'imposer ? S'agissant de cette dérivation apophonique, on serait tenté de faire valoir que l'énorme stock d'adjectifs en -oso, exclus du corpus, joue nécessairement un rôle dans la productivité de celle-ci: si l'on ajoute ces 283 adjectifs aux autres formes apophoniques, ils constituent, en effet, près de $80 \%$ de la classe et plus de $88 \%$ des pluriels apophoniques analogiques! Il est certain que la prise en compte des adjectifs en -oso changerait radicalement les données du problème, lequel, du coup, n'en serait plus un, puisque, le nombre de formes apophoniques (355) dépassant alors de plus $\mathrm{du}$ double celui des formes non apophoniques (166), la productivité d'une dérivation serait, dans cette hypothèse, corrélée à sa fréquence lexicale. Je suis prêt à admettre que l'annexion de ce suffixe hautement productif ${ }^{11}$ par la classe apophonique $^{12}$ a dû être pour beaucoup dans la survie de

11. Conformément à l'observation de Hock rapportée plus haut, je fais ici allusion non pas tant au nombre d'adjectifs en -oso qu'au fait qu'ils constituent une classe ouverte, une grande partie d'entre eux devant donc être sémantiquement transparents.

12. Cette désinence, apophonique (-[0]sos) bien qu'issue de lat. -ōsu et correspondant à cast. -oso, est, en effet, analogique. Son annexion par la classe apophonique a probablement été favorisée par la métaphonie du féminin, l'influence ouvrante de $-a$ sur /o/ ( $c f$. n. 2) étant attestée dès l'époque médiévale ( $c f$. Huber 1933 : $\S 98$ ). A preuve, le même suffixe est resté non apophonique dans les noms propres (Barroso, Cardoso, 


\section{J. BRANDÃO DE CARVALHO}

l'alternance [o] - [0], alors même que de nombreux mots, aujourd'hui apophoniques dans le standard lisboète, présentaient sans doute un $/ 0 /$ invariable il y a à peine deux siècles. Elle ne me semble pourtant pas pouvoir expliquer, à elle seule, le renversement de la tendance analogique.

Si l'ensemble des adjectifs en -oso devait être intégré au corpus, alors de deux choses l'une : il nous faut soit admettre qu'ils sont tous dans le lexique, soit soutenir, à l'instar de Bybee (2001) ou de Pierrehumbert (2002), que les facteurs pertinents pour l'analyse sont d'ordre fonctionnel et reposent sur la fréquence d'usage de chaque output, ou plutôt, le concept d' «entrée lexicale» n'ayant plus guère de sens dans cette perspective, sur celle de chaque « exemplaire » (token).${ }^{13}$ Dans le premier cas de figure, si tous les adjectifs en -oso étaient dans le lexique, ce qui poserait alors problème serait la « résistance » qu'offre à l'analogie la dérivation non apophonique, du coup nettement minoritaire : après tout, comme je l'ai signalé plus haut, la plupart des emprunts font leur pluriel en /o/. D'autre part, toujours dans cette hypothèse, on ne comprendrait pas pourquoi la dérive attestée dans le standard lisboète ne revêt pas la même ampleur dans tous les dialectes portugais, notamment au Brésil, où -oso est aussi apophonique. Dans le second cas, celui où seule compterait la fréquence d'usage des tokens, je vois mal comment on pourrait empiriquement déterminer des différences statistiques susceptibles d'expliquer, encore une fois, la divergence entre le standard lisboète et d'autres variétés de portugais: fréquence d'usage, soit, mais de quels usages

Fragoso, Matoso, Pedroso, etc. - non comptabilisés dans le corpus) en l'absence de féminin.

13. On peut certes opter pour une solution de compromis, en faisant l'hypothèse que seuls certains des adjectifs en -oso sont lexicaux, ou bien encore que, le lexique constituant un "ensemble flou» et l'appartenance à celui-ci obéissant à un gradient, ces adjectifs sont plus ou moins lexicaux. Mais se pose alors le problème des critères de décision : à quoi tiendrait le statut (plus ou moins) lexical de tel ou tel adjectif? A la complexité phonologique de sa dérivation (maldoso) ? A l'opacité sémantique de son radical (jeitoso, curioso) ? A sa fréquence d'usage (gostoso) ? Je laisse ces points en suspens. Ils impliquent une autre étude de corpus, cette fois celle des formes en -oso, mais surtout une réponse aux questions théoriques ci-dessus. 


\section{L' analogie est-elle un fait fonctionnel ou grammatical?}

parle-t-on? De plus, indépendamment des problèmes inhérents à l'établissement d'un tel corpus, on sait que, s'il est vrai que la fréquence d'usage d'un mot favorise le changement phonétique (cf. Wang 1977), les choses sont nettement moins claires s'agissant des processus analogiques : les verbes dits irréguliers, c'est-à-dire préservés de toute réfection analogique, sont aussi, précisément, les plus fréquents.

\section{Conclusion}

L'explication de l'expansion de l'apophonie dans la língua padrão européenne semble donc relever de facteurs autres que statistiques, qu'il s'agisse de la taille des classes ou de la fréquence d'usage des types ou des exemplaires. Deux remarques à ce sujet, l'une d'ordre empirique, l'autre théorique. La première est que, quelle qu'en soit l'explication, les faits portugais illustrent bien les limites d'une analyse de corpus purement ahistorique. Au mieux, le relevé systématique des mots soumis à l'une ou l'autre de ces alternances, puis le calcul de l'importance numérique relative des divers groupes lexicaux ne nous apprennent rien, à eux seuls, sur la lente dérive en cours depuis au moins deux siècles; au pire, ils risquent fort de nous induire en erreur, compte tenu de la prédominance arithmétique de la classe non apophonique. Si ces données, par ailleurs indispensables, ne sont pas situées dans une perspective historique et confrontées à un paramètre étymologique, ainsi que je me suis efforcé de le faire, alors il ne nous est guère possible de déterminer la dynamique en jeu et le sens de l'évolution, et donc de nous apercevoir qu'il y a là un problème pour la théorie diachronique.

Le second point, cette fois d'ordre théorique, sur lequel je voudrais insister et qui demande de plus amples développements, est que l'existence de tels problèmes est nécessaire au regard de ce que l'on sait de l'évolution linguistique. Si la fréquence d'une dérivation découlait systématiquement de sa productivité, et que, réciproquement, celle-ci était étayée par celle-là, alors, l'une entretenant l'autre ad ceternum, on voit mal ce qui pourrait faire bouger les choses. Or il est un fait avéré qu'une alternance finit, tôt ou tard, par être remplacée. Si l'on 


\section{J. BRANDÃO DE CARVALHO}

exclut les facteurs statistiques, quelles sont donc les causes de la dérive portugaise en cours?

S'agissant de ses causes contingentes et spécifiques, qui distinguent le parler lisboète des autres variétés de portugais, tant le caractère récent de cette évolution que le fait qu'elle favorise l'alternance la plus complexe permettent d'y supposer certains des traits sociolinguistiques qu'Encrevé (1988: § 5) avait décelés dans le retour en force de la liaison en français contemporain : influence croissante d'un discours "scolaire » sur la norme, ${ }^{14}$ développement d'un sentiment d'insécurité linguistique - et, partant, d'une tendance à l'hypercorrection en rapport avec l'accroissement de la mobilité sociale, parmi d'autres facteurs.

Cependant, le fait même qu'un ensemble de locuteurs puisse investir d'une valeur sociolinguistique donnée une alternance lexicalement minoritaire tend à montrer que le facteur potentiellement déclenchant de son expansion réside dans la sensibilité à un schème dérivationnel, perçu, dans ce cas d'espèce, comme concurrent du schème majoritaire, tous deux se partageant le même ensemble lexical ( $c f$. § 4). Ce point est intéressant en ce qu'il touche à la nature des processus dits analogiques. L'analogie fut primitivement conçue en termes de rapports entre "groupes de formes" (cf. Paul 1966 [1920]: $\S 5)$; cette conception survit aujourd'hui tant dans la mouvance néo-générative ( $c f$., par exemple, la théorie des «output-output correspondence constraints » défendue par Burzio 1996) que dans les approches fonctionnalistes (cf. Bybee 2001). Dans les trois cas, le «modèle » suivi par le changement analogique est, en quelque sorte, incarné dans les formes directement offertes à l'interprétation, à la fameuse "activité créatrice», du locuteur. Il découle de telles vues que l'analogie se situe en dehors de la grammaire conçue comme impliquant la mise en œuvre inconsciente d'une faculté spécifique.

Or le problème posé par les faits portugais suggère, au contraire, que les «groupes de formes» de Paul - ces

14. Encore qu'il n'y ait pas ici de facteur orthographique comparable, les graphies avec $\hat{o}$ n'ayant jamais été systématiquement suivies, surtout au Portugal. 


\section{L' analogie est-elle un fait fonctionnel ou grammatical?}

ensembles de lexèmes réunis par un lien morphophonologique - ne sont pas, par eux-mêmes, le moteur des phénomènes de propagation analogique; ils n'en sont que l'expression, souvent instable et passagère, où, comme on l'a $\mathrm{vu}$, un mot étymologiquement apophonique peut être annexé par l'ensemble rival pour revenir plus tard à sa classe originelle. A l'image des vagues, dont la direction n'implique nullement un mouvement parallèle des molécules d'eau, mais s'explique par une force qui parcourt la matière liquide, je soutiens que ce sont de purs rapports dérivationnels qui structurent le lexique. Ce n'est pas le couple sing / sang ni même l'ensemble des doublets du même type qui induisent bring / brang; c'est le "schème -ing / -ang" qui est responsable des formes étymologiques comme des formes analogiques. C'est donc à l'existence de tels schèmes que l'on doit notamment la productivité (éventuellement croissante) d'alternances minoritaires, ce que ne sauraient expliquer, on l'a vu, les approches statistiques fondées sur les items lexicaux. ${ }^{15}$. L'hypothèse de schèmes dérivationnels, abstraits du lexique qu'ils contribuent à structurer, implique que les processus analogiques font indiscutablement partie de la grammaire, au même titre que les processus phonologiques et syntaxiques classiques. Les faits portugais, énigmatiques dès lors qu'on les appréhende par les sorties lexicales, s'expliquent si on les envisage en termes de concurrence entre deux schèmes qui, à l'inverse de -ing / -ang ou -que / -cité, ne sont pas isolés dans leur domaine phonologique respectif. Ces faits constituent ainsi un argument empirique majeur à l'appui de l'hypothèse de tels schèmes, et, par là, du caractère grammatical de l'analogie.

15. Et c'est aussi à l'existence de tels schèmes que l'on doit, notons-le, la productivité d'alternances qui n'ont, depuis longtemps, plus aucune assise phonologique : on sait, par exemple, que la transformation, en français, de $/ \mathrm{k} /$ en /s/ devant /i/ dans opaque / opacité, électrique / électricité, etc. est productive face à l'absence d'allomorphie dans antique / antiquité, et ce bien que /s/ ne soit pas une consonne palatale. 


\section{J. BRANDÃO DE CARVALHO}

\section{Références bibliographiques}

Burzio L. (1996). «Surface constraints versus underlying representation ", in J. Durand \& B. Laks (éds.) Current trends in phonology: Models and methods. Vol. 1. Salford: University of Salford, European Studies Research Institute, $125-144$.

Bybee J. (2001). Phonology and language use. Cambrigde: Cambridge University Press.

Carvalho J. Brandão de (1994). «What are vowels made of ? The 'no-rule' approach and particle phonology », Studia linguistica $48: 1-27$.

Carvalho J. Brandão de (2004). «Templatic morphology in the Portuguese verb », in T. Meisenburg \& M. Selig (éds.) Nouveaux départs en phonologie. Tübingen: Gunter Narr Verlag, 13-32.

DLPC (2001). Dicionário da língua portuguesa contemporânea da Academia das Ciências de Lisboa. Lisbonne : Verbo.

Encrevé P. (1988). La liaison avec et sans enchaînement: Phonologie tridimensionnelle et usages du français. Paris : Seuil.

Ferreira A. Buarque de Hollanda (1977). Minidicionário Aurélio. Rio de Janeiro : Nova Fronteira.

Hock H. H. (1988). Principles of historical linguistics. Berlin : Mouton-De Gruyter.

Huber J. (1933). Altportugiesisches Elementarbuch. Heidelberg : Carl Winters.

Nunes J. J. (1945). Compêndio de gramática histórica portuguesa. $3^{\mathrm{e}}$ éd. Lisbonne : Livraria Clássica.

Paul, H. (1966). Prinzipien der Sprachgeschichte. Tübingen : Max Niemeyer Verlag. [Réimpression de la $5^{\text {ème }}$ éd. de 1920.]

Pierrehumbert J. B. (2002). Probabilistic phonology: Dicrimination and robustness. Ms., Northwestern University, Evanston, Il.. 
L' analogie est-elle un fait fonctionnel ou grammatical?

Russo M. (2001). La métaphonie en napolitain: Evolution et fonctionnement synchronique. Thèse de doctorat, Université de Paris 8.

Wang W. S.-Y. (1977) (éd.). The lexicon in phonological change. La Haye : Mouton.

Williams E. B. (1938). From Latin to Portuguese: Historical phonology and morphology of the Portuguese language. Philadelphie : University of Pennsylvania Press. 


\section{Annexes}

\section{A. Formes apophoniques : 72 items}

\section{A.1 Formes apophoniques analogiques : 38 items}

$\begin{array}{llll}\text { abrolho } & \text { tribule, épines } & \text { folho } & \text { jabot } \\ & \text { forno } & \text { four } \\ \text { almoço } & \text { déjeuner } & \text { jorro } & \text { jaillissement } \\ \text { antolho } & \text { illusion, envie, } & \text { miolo } & \text { cervelle, mie } \\ & \text { eillères (pl.) } & \text { molho } & \text { sauce } \\ \text { bolso } & \text { poche } & \text { morno } & \text { tiède } \\ \text { caroço } & \text { noyau } & \text { olho } & \text { œil } \\ \text { choco } & \text { seiche } & \text { pescoço } & \text { cou } \\ \text { contorno } & \text { contour } & \text { poço } & \text { puits } \\ \text { corcovo } & \text { courbe } & \text { rebordo } & \text { rebord } \\ \text { despojo } & \text { dépouille } & \text { reforço } & \text { renfort } \\ \text { desporto } & \text { sport } & \text { sobrolho } & \text { sourcil } \\ \text { destroço } & \text { débris } & \text { socorro } & \text { secours } \\ \text { escolho } & \text { écueil } & \text { tojo } & \text { ajonc } \\ \text { esforço } & \text { effort } & \text { tordo } & \text { grive } \\ \text { esgoto } & \text { égoût } & \text { torno } & \text { tour, étau } \\ \text { esposo } & \text { époux } & \text { torso } & \text { torse } \\ \text { estofo } & \text { étoffe } & \text { tremoço } & \text { lupin } \\ \text { estofo } & \text { étale (adj.) } & \text { troço } & \text { tronçon } \\ \text { estojo } & \text { étui } & \text {-oso } & \text {-eux }\end{array}$

\section{A.2 Formes apophoniques étymologiques : 34 items}

$\begin{array}{llll}\text { choco } & \text { couvaison, couvé } & \text { horto } & \text { potager } \\ \text { corno } & \text { corne } & \text { imposto } & \text { impôt } \\ \text { coro } & \text { chœur } & \text { jogo } & \text { jeu } \\ \text { corpo } & \text { corps } & \text { morto } & \text { mort } \\ \text { corvo } & \text { corbeau } & \text { novo } & \text { nouveau } \\ \text { dorminhoco } & \text { qui dort beaucoup } & \text { osso } & \text { os } \\ \text { envolto } & \text { entouré } & \text { ovo } & \text { cuf } \\ \text { fogo } & \text { feu } & \text { paradoxo } & \text { paradoxe } \\ \text { foro } & \text { droit } & \text { polvo } & \text { pieuvre } \\ \text { fosso } & \text { fossé } & \text { porco } & \text { sale } \\ \text { grosso } & \text { gros } & \text { porco } & \text { porc }\end{array}$


L' analogie est-elle un fait fonctionnel ou grammatical?

$\begin{array}{llll}\text { porto, } & \text { port, } & \text { revolto } & \text { agité } \\ \text { aeroporto... } & \text { aéroport... } & \text { rogo } & \text { prière } \\ \text { posto } & \text { poste } & \text { sogro } & \text { beau-père } \\ \text { posto, etc. } & \text { mis } & \text { tijolo } & \text { brique } \\ \text { povo } & \text { peuple } & \text { torto } & \text { tordu } \\ \text { recosto } & \text { pente } & \text { troco } & \text { monnaie } \\ \text { renovo } & \text { rejeton } & & \end{array}$

\section{B. Formes non apophoniques : 166 items}

\section{B.1 Formes non apophoniques analogiques : 40 items}

\begin{tabular}{|c|c|c|c|}
\hline aborto & avortement & entrecosto & plat de côtes \\
\hline absorto & absorbé & escopo & but, intention \\
\hline acordo & accord & gafanhoto & sauterelle \\
\hline alho-porro & poireau & garoto & gamin \\
\hline bacoco & niais & globo & globe \\
\hline barroco & perle & goro & œuf non fécondé \\
\hline bicharoco & bestiole & maroto & polisson \\
\hline canhoto & gaucher & minhoto & du Minho \\
\hline carolo & épi de maïs & molosso & molosse \\
\hline cocho & sorte de récipient & mormo & morve, maladie \\
\hline colosso & colosse & & des chevaux \\
\hline covo & creux & oco & creux \\
\hline denodo & audace & passaroco & oiseau (dim.) \\
\hline desacordo & désaccord & perdigoto & perdreau \\
\hline desaforo & insolence & probo & probe \\
\hline desenvolto & désinvolte, adroit & recordo & souvenir \\
\hline dolo & tromperie & soldo & solde \\
\hline dorso & dos & soro & petit-lait \\
\hline encosto & dossier (dun & sorvo & gorgée \\
\hline & siège) & torvo & sinistre \\
\hline endosso & endossement & tropo & trope \\
\hline
\end{tabular}

\section{B.2 Formes non apophoniques étymologiques : 126 items}

$\begin{array}{llll}\text { aboio } & \text { sorte de chant } & \text { antojo, entojo } & \text { dégoût } \\ \text { adorno } & \text { ornement } & \text { apodo } & \text { moquerie } \\ \text { agosto } & \text { août } & \text { apoio } & \text { appui } \\ \text { alvoroço } & \text { agitation } & \text { apojo } & \text { lait gras }\end{array}$




\section{J. BRANDÃO DE CARVALHO}

\begin{tabular}{|c|c|c|c|}
\hline arroio & ruisseau & emboço & crépi \\
\hline arrojo & audace & esboço & ébauche \\
\hline arroto & rot & escopro & ciseau \\
\hline bobo & bouffon & escorço & raccourci (pict.) \\
\hline bobo & sot & escroto & scrotum \\
\hline bodo & $\begin{array}{l}\text { repas donné aux } \\
\text { pauvres }\end{array}$ & estorço & $\begin{array}{l}\text { attitude contrainte } \\
\text { (peint.) }\end{array}$ \\
\hline bojo & ventre, saillie & estorno & ristourne \\
\hline bolbo & bulbe & estorvo & gêne \\
\hline bolo & gâteau & estropo & cordage \\
\hline borro & agneau & ferrolho & verrou \\
\hline boto & émoussé & filantropo, & philanthrope, \\
\hline broto & bourgeon & misantropo & misanthrope \\
\hline caboclo & métis & fofo & moelleux \\
\hline cachorro & chiot & forro & doublure \\
\hline cebolo & oignon & forro & affranchi \\
\hline cerefolho & cerfeuil & fosco & obscur \\
\hline chocho & desseché & Godo & Goth \\
\hline choro & pleurs & golfo & golfe \\
\hline coco & noix de coco & golo & but (foot) \\
\hline cofo & panier & golo & gorgée \\
\hline colmo & chaume & gordo & gras \\
\hline conforto & confort & gorro & bonnet \\
\hline consolo & consolation & gosto & goût \\
\hline corço & jeune cerf & goto & gosier \\
\hline corso & course & gozo & plaisir \\
\hline corso & corse & ignoto & inconnu \\
\hline coto & bout & insosso & fade \\
\hline coxo & boîteux & joio & ivraie \\
\hline demagogo, & démagogue, & lobo & loup \\
\hline pedagogo & pédagogue & lodo & vase \\
\hline desafogo & soulagement & logro & tromperie \\
\hline descoco & bêtise & loto & loto \\
\hline desconsolo & désolation & malogro & échec \\
\hline desembolso & déboursement & mocho & hibou \\
\hline \multirow[t]{2}{*}{ desforço } & réparation (pour & mocho & écorné \\
\hline & une offense) & moco & morve \\
\hline desgosto & chagrin & moço & jeune \\
\hline dobro & double & mofo & moisissure \\
\hline
\end{tabular}


L' analogie est-elle un fait fonctionnel ou grammatical?

$\begin{array}{llll}\text { morro } & \text { tertre } & \text { rolho } & \text { trapu } \\ \text { mosto } & \text { moût } & \text { rolo } & \text { rouleau } \\ \text { namoro } & \text { flirt } & \text { rosto } & \text { visage } \\ \text { nojo } & \text { dégoût } & \text { rostro } & \text { bec } \\ \text { olmo } & \text { orme } & \text { roto } & \text { déchiré } \\ \text { piloto } & \text { pilote } & \text { roxo } & \text { violet } \\ \text { pimpolho } & \text { bourgeon } & \text { salobro } & \text { saumâtre } \\ \text { piolho } & \text { pou } & \text { saloio } & \text { saloio, plouc } \\ \text { piropo } & \text { propos galant } & \text { soco } & \text { coup de poing } \\ \text { pojo } & \text { banc de pierre } & \text { soçobro } & \text { naufrage } \\ \text { poldro } & \text { poulain } & \text { solho } & \text { esturgeon } \\ \text { potro } & \text { poulain } & \text { solto } & \text { lâché } \\ \text { raposo } & \text { renard } & \text { sopro, assopro } & \text { souffle } \\ \text { rebolo } & \text { meule à affûter } & \text { suborno } & \text { corruption } \\ \text { redobro } & \text { redoublement } & \text { todo } & \text { tout } \\ \text { reembolso } & \text { remboursement } & \text { toldo } & \text { bâche } \\ \text { repolho } & \text { choux } & \text { topo } & \text { sommet } \\ \text { restolho } & \text { éteule } & \text { tosco } & \text { grossier } \\ \text { retorno } & \text { retour } & \text { trambolho } & \text { embarras } \\ \text { risoto } & \text { risotto } & \text { transbordo } & \text { transbodement } \\ \text { rodo } & \text { râteau } & \text { transtorno } & \text { dérangement } \\ \text { rojo } & \text { action de traîner } & \text { zarolho } & \text { borgne } \\ & & & \end{array}$

C. Formes à étymologie inconnue : 9 items

\section{C.1 Formes apophoniques : 1 item}

cachopo garçon, récif

\section{C.2 Formes non apophoniques : 8 items}

$\begin{array}{llll}\text { arrocho } & \text { gourdin } & \text { toco } & \text { souche, bout } \\ \text { balofo } & \text { bouffi } & \text { tolo } & \text { sot } \\ \text { carocho } & \text { scarabée } & \text { xarroco, } & \text { baudroie } \\ \text { engodo } & \text { appât } & \text { enxarroco } & \\ \text { reboco } & \text { crépi } & & \end{array}$

